ESAIM: PROCEEDINGS, December 2013, Vol. 42, p. 1-9

A. Heibig, F. Filbet, L. I. Palade, Editors

\title{
VARIFOLDS AND GENERALIZED CURVATURE
}

\author{
BlANCHE BuET $^{1}$
}

\begin{abstract}
This short note aims at introducing, with a few simple examples, the notions of varifold and generalized mean curvature.

Résumé. Notre objectif est d'aborder les notions de varifold et de courbure gnralise au travers d'exemples simples.
\end{abstract}

\section{WHAT IS A VARIFOLD?}

\subsection{Context}

Varifolds were introduced by F. Almgren in [2] as he was interested in solving Plateau's problem in a broader class of surfaces. Roughly, Plateau's Problem can be formulated in the following form

Problème 1.1 (Plateau's Problem). Given a closed curve $\Gamma$ in $\mathbb{R}^{3}$, is there a surface with minimal area spanning it ?

Obviously, this problem depends on the hypothesis on $\Gamma$, the set of surfaces one authorizes and the meaning one gives to span. In 1931 (cf. [5]), J. Douglas solved the problem in the restricted class of surfaces being parametrized images of a disk but this excludes the case of non-orientable surfaces or triple-junction. The problem is that Mœbius strip or junctions can be obtained as soap films. Almgren introduced varifolds to tackle Plateau's Problem in a class containing such surfaces. A varifold is a generalized surface, which can be non-orientable and which can contain singularities such as junctions. The class of varifolds is endowed with notions of generalized curvature, convergence and compactness properties. Nevertheless, defining the boundary of a varifold is still an issue.

\subsection{Some examples}

So as to understand why Almgren introduced varifolds we can bring out two main ingredients of this notion.

- First, when working with varifolds, one works with measures instead of surfaces or manifolds and one has then a convenient notion of convergence which is the convergence in the sense of measures. For instance, if $S \subset \mathbb{R}^{3}$ is a surface, an obvious way of associating a measure to $S$ is to consider the measure $\mu:=\mathcal{H}_{\mid S}^{2}$ on $\mathbb{R}^{3}$, where $\mathcal{H}^{d}$ denote the $d$-dimensional Hausdorff measure, and to recover $S$ from $\mu$ by taking the support of $\mu$. One could also consider any measure of the form $\theta \mathcal{H}_{\mid S}^{2}$ with $\theta: S \rightarrow \mathbb{R}_{+}$. But these measures are not varifolds associated to $S$, they are only part of the information required to define a varifold associated to $S$. A varifold contains more information about $S$, this leads us to the the second point.

1 Institut Camille Jordan, Universit Claude Bernard Lyon 1, 43 boulevard du 11 novembre 1918, 69622 VILLEURBANNE Cedex

(C) EDP Sciences, SMAI 2013 

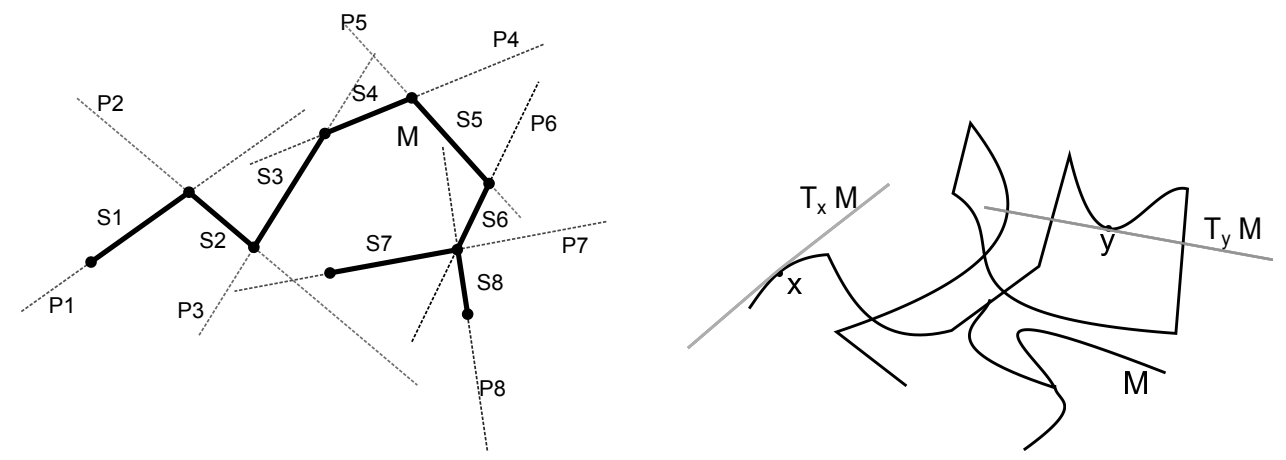

Figure 1. Polygonal curve and rectifiable curve

- A varifold associated to the surface $S$ combines information both on position and tangent plane (while the measure $\mu=\mathcal{H}_{\mid S}^{2}$ gives only position). Actually, the object "position + tangent plane" we want to consider is exactly $T S:=\left\{\left(x, T_{x} S\right) \mid x \in S\right\}$ with $T_{x} S$ the tangent plane to $S$ at the point $x$. Thus a varifold will be a measure whose support is $T S$. Thanks to this additional information of tangent plane, one can define a notion of generalized curvature in the class of varifolds and such that if a sequence of varifolds converges to a varifold then the curvature converges too (in a weak sense).

Now, if $M \subset \mathbb{R}^{n}$ is a $d$-submanifold then $T M:=\left\{\left(x, T_{x} M\right) \mid x \in M\right\} \subset \mathbb{R}^{n} \times G_{d, n}$ with $G_{d, n}$ being the set of all vector subspaces of dimension $d$ in $\mathbb{R}^{n}$. Therefore a varifold associated to $M$ is a Radon measure on $\subset \mathbb{R}^{n} \times G_{d, n}$ and the canonical varifold associated to $M$ is the Radon measure $\mathcal{H}_{\mid M}^{d} \otimes \delta_{T_{x} M}$ on $\mathbb{R}^{n} \times G_{d, n}$. We explain the notation $\mathcal{H}_{\mid M}^{d} \otimes \delta_{T_{x} M}$ in Definition 1.6, but first we focus on some simple examples.

Exemple 1.2. Consider a straight line $D \subset \mathbb{R}^{2}$, then the measure $v(D)=\mathcal{H}_{\mid D}^{1} \otimes \delta_{D}$ is the canonical varifold associated to $D$. Thus,

- for every Borel set $B \subset \mathbb{R}^{2} \otimes G_{1,2}$,

$$
v(D)(B)= \begin{cases}\mathcal{H}^{1}\left(\pi_{1}(B)\right) & \text { si } D \in \pi_{2}(B) \\ 0 & \text { sinon }\end{cases}
$$

where $\pi_{1}: \mathbb{R}^{2} \otimes G_{1,2} \rightarrow \mathbb{R}^{2},(x, T) \mapsto x$ and $\pi_{2}: \mathbb{R}^{2} \otimes G_{1,2} \rightarrow G_{1,2},(x, T) \mapsto T$ are the projections onto $\mathbb{R}^{2}$ and $G_{1,2}$,

- for every continuous compactly supported function $\varphi \in C_{c}\left(\mathbb{R}^{2} \times G_{1,2}, \mathbb{R}\right)$,

$$
\int_{\mathbb{R}^{2}} \int_{G_{1,2}} \varphi(x, T) d v(D)(x, T)=\int_{\mathbb{R}^{2}} \varphi(x, D) d \mathcal{H}_{\mid D}^{1}(x)
$$

Exemple 1.3. Consider a polygonal curve $M \subset \mathbb{R}^{2}$ consisting of 8 line segments $S_{1}, \ldots, S_{8}$ of direction $P_{1}, \ldots, P_{8} \in G_{1,2}$, then the measure $v(M)=\sum_{i=1}^{8} \mathcal{H}_{\mid S_{i}}^{1} \otimes \delta_{P_{i}}$ is the canonical varifold associated to $M$.

Exemple 1.4. Consider now a rectifiable curve $M \subset \mathbb{R}^{2}$ and denote $T_{x} M \in G_{1,2}$ its tangent at a point $x \in M$. As before, we want to define the measure $v(M)=\mathcal{H}_{\mid M}^{1} \otimes \delta_{T_{x} M}$, which can be done by

- for every Borel subset $B \subset \mathbb{R}^{2} \times G_{1,2}, v(M)(B)=\mathcal{H}^{1}\left(\pi_{1}(T M \cap B)\right)$ where $\pi_{1}$ is the projection onto $\mathbb{R}^{2}$ as previously; or equivalently,

- for every continuous compactly supported function $\varphi \in C_{c}\left(\mathbb{R}^{2} \times G_{1,2}, \mathbb{R}\right)$,

$$
\int_{\mathbb{R}^{2}} \int_{G_{1,2}} \varphi(x, T) d v(M)(x, T)=\int_{\mathbb{R}^{2}} \varphi\left(x, T_{x} M\right) d \mathcal{H}_{\mid M}^{1}(x)
$$


Exemple 1.5 (Adding density). In all the previous examples, we used the Hausdorff measure to measure the space but it is possible to use $\theta \mathcal{H}_{\mid M}^{1}$ instead, where $\theta: M \rightarrow \mathbb{R}_{+}^{*} \in \mathrm{L}_{l o c}^{1}\left(M, \mathcal{H}^{1}\right)$ is called the multiplicity of the varifold. For instance, in Example 1.2, one can define the varifold $v(D, \theta)=\theta \mathcal{H}^{1} \otimes \delta_{D}$ and in Example 1.3, one can define the varifold $v(M, \theta)=\sum_{i=1}^{8} \theta_{i} \mathcal{H}_{\mid S_{i}}^{1} \otimes \delta_{P_{i}}$, with $\theta=\theta_{i}$ on $S_{i}$. As $\theta>0$ on $M$, the varifolds $v(M)=v(M, 1)$ and $v(M, \theta)$ have same support $T M=\left\{\left(x, T_{x} M\right), x \in M\right\}$ thus by adding a multiplicity $\theta$, one changes only the weigth of $M$. If one gives multiplicity $\theta=2$ everywhere on $M$ to a varifold, it amounts to "counting twice" $M$.

Now, we can define the notion of varifold.

\subsection{Definition of a varifold}

First, we define the notion of a $d$-rectifiable varifold.

Définition 1.6. Let $\Omega \subset \mathbb{R}^{n}$ be an open subset. A $d$-rectifiable varifold $V$ in $\Omega$ is a measure on $\Omega \times G_{d, n}$ of the form

$$
\begin{gathered}
V=\theta \mathcal{H}_{\mid M}^{d} \otimes \delta_{T_{x} M} \\
\text { i.e. } \int_{\Omega \times G_{d, n}} \varphi(x, T) d V(x, T)=\int_{M} \varphi\left(x, T_{x} M\right) \theta(x) d \mathcal{H}^{d}(x) \quad \forall \varphi \in C_{c}\left(\Omega \times G_{d, n}, \mathbb{R}\right) \\
\text { i.e. } V(B)=\int_{\pi_{1}(T M \cap B)} \theta d \mathcal{H}^{d} \quad \forall B \subset \Omega \otimes G_{d, n} \text { Borel }
\end{gathered}
$$

where

- $M$ is a $d$-dimensional rectifiable subset of $\Omega$ (see [6]),

- $T_{x} M$ is the approximative tangent space at $x$ which exists $\mathcal{H}^{d}$-almost everywhere in $M$ and $T M=$ $\left\{\left(x, T_{x} M\right) \mid x \in M\right\}$,

- $\theta$ is a positive locally $\mathcal{H}^{d}$-summable function on $M$,

- $\pi_{1}: \Omega \otimes G_{d, n} \rightarrow \Omega$ is the projection onto $\Omega$.

Remarque 1.7. We are dealing with measures on $\Omega \times G_{d, n}$, but we did not mention the $\sigma$-algebra we consider. We can equip $G_{d, n}$ with the metric

$$
d(T, P)=\left\|\Pi_{T}-\Pi_{P}\right\|
$$

with $\Pi_{T} \in M_{n}(\mathbb{R})$ being the matrix of the orthogonal projection onto $T$ and $\|\cdot\|$ any norm on $M_{n}(\mathbb{R})$. We consider measures on $\Omega \times G_{d, n}$ with respect to the Borel algebra on $\Omega \times G_{d, n}$.

Définition 1.8 (Varifold). A $d$-varifold in $\Omega$ (open subset of $\mathbb{R}^{n}$ ) is a positive Radon measure on $\Omega \times G_{d, n}$.

Remarque 1.9. As $\Omega \times G_{d, n}$ is locally compact, a positive Radon measure on $\Omega \times G_{d, n}$ is just a Borel measure finite on compact sets. Thus, any Radon measure $V$ on $\Omega \times G_{d, n}$ naturally defines a continuous positive linear map

$$
C_{c}\left(\Omega \times G_{d, n}\right) \rightarrow \mathbb{R}, \quad \varphi \mapsto \int_{\Omega \times G_{d, n}} \varphi(x, P) d V(x, P)
$$

Conversely, by Riesz Theorem, we can identify Radon measures on $\Omega \times G_{d, n}$ and linear forms on $C_{c}\left(\Omega \times G_{d, n}\right)$.

Exemple 1.10. Consider the plane $P=\{z=0\}$ in $\mathbb{R}^{3}$. One can associate to this plane the canonical 2varifold $V_{1}=\mathbb{H}_{\mid P}^{2} \otimes \delta_{P}$ but one can also build the 1-varifold $V_{2}=\mathbb{H}_{\mid P}^{2} \otimes \delta_{D}$ with $D \subset P$ a straight line. $V_{1}$ is a 2 -rectifiable varifold while $V_{2}$ is a 1 -varifold, but is not 1 -rectifiable (since $P$ is an object of dimension 2 it can not be 1-rectifiable).

Conversely, it is possible (in some cases) to build a $n$-varifold from a $d$-varifold in $\mathbb{R}^{n}$, for instance: 

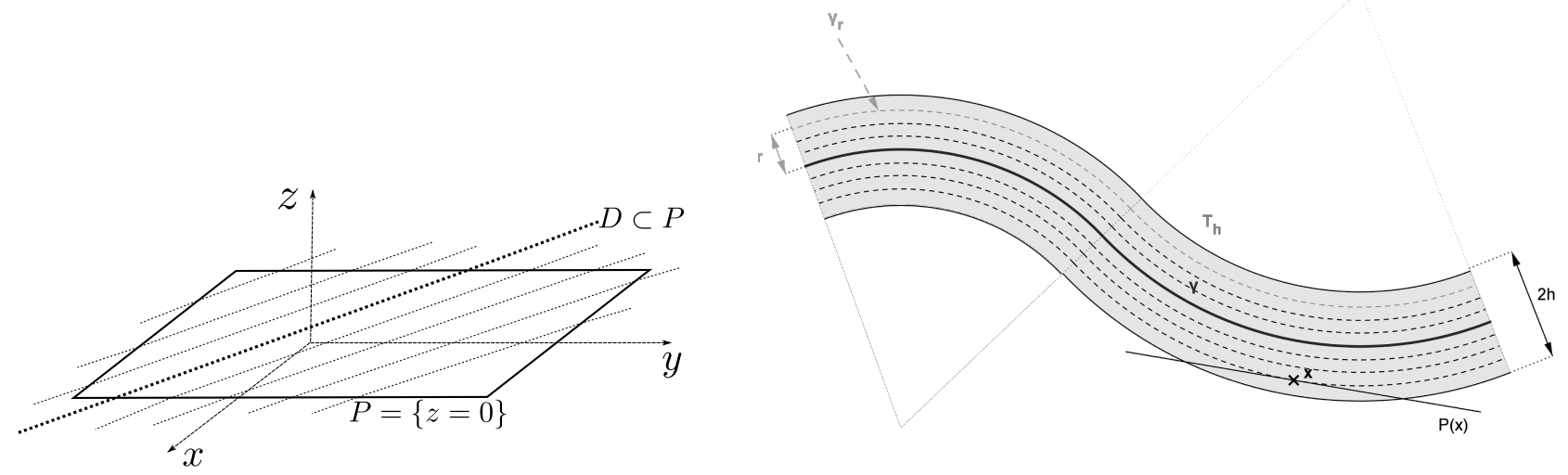

FiguRE 2. Diffuse varifolds

Exemple 1.11. Take a smooth curve $\gamma$ and let $d$ denote the signed distance function to $\gamma$. We call $\gamma_{r}$ the $r$-level line of $\gamma$. Let $h$ be such that $d$ is well defined in a $h$-tubular neighbourhood $T_{h}=\{x \mid d(x, \gamma) \leq h\}$ of $\gamma$. We can now define the diffuse varifold $v_{\gamma}$ such that for every $\varphi \in C_{c}\left(\mathbb{R}^{n} \times G_{d, n}\right)$,

$$
\int \varphi(x, S) d v_{\gamma}(x, S)=\int_{T_{h}} \varphi\left(x, T_{\pi(x)} \gamma\right) d x
$$

with $\pi: T_{h} \rightarrow \gamma$ the projection onto $\gamma$.

\section{Curvature of a varifold}

\subsection{First variation of the area functional and the divergence theorem}

The area functional $\mathcal{A}$ in $\Omega$ is defined as

$$
\mathcal{A}(M)=\mathcal{H}^{d}(M)
$$

for every $M$ being a $d$-submanifold of $\Omega \subset \mathbb{R}^{n}$. Now, given any vector field $X \in C_{c}^{1}\left(\Omega, \mathbb{R}^{n}\right)$, let $\phi_{t}(x)=x+t X(x)$. For $t$ small enough, $\phi_{t}$ is a diffeomorphism of $\Omega$. $M$ is said to be a critical point of the functional area if for every $X \in C_{c}^{1}\left(\Omega, \mathbb{R}^{n}\right)$,

$$
\left.\frac{d}{d t} \mathcal{A}\left(\phi_{t}(M)\right)\right|_{t=0}=0
$$

Now, with some computations (using the coarea formula and the divergence theorem, see [8]), one has

$$
\left.\frac{d}{d t} \mathcal{A}\left(\phi_{t}(M)\right)\right|_{t=0}=\int_{M} \operatorname{div}_{M} X(x) d \mathcal{H}^{d}(x)=-\int_{M} H \cdot X d \mathcal{H}^{d}
$$

$H$ denotes the mean curvature vector and the differential operator $\operatorname{div}_{M}$ is defined by

$$
\operatorname{div}_{M} X(x)=\sum_{j=1}^{d}\left\langle\tau_{j}, D X(x) \cdot \tau_{j}\right\rangle
$$

with $\left(\tau_{1}, \ldots, \tau_{d}\right)$ an orthonormal basis of the tangent plane $T_{x} M$. Now, we can recall a famous fact: 
Proposition 2.1. $M$ is a critical point of the area functional if and only if its mean curvature is zero everywhere. Such a surface is called a minimal surface.

Remarque 2.2. Of course, any critical point is not a minimum of the functional area but is nevertheless called a minimal surface.

Same kind of arguments holds for rectifiable varifolds so that, if $V=v(M, \theta)$ is a $d$-rectifiable varifold and $\mathcal{A}(V)=\int_{M} \theta(x) d \mathcal{H}^{d}(x)$ denotes the area functional, one has

$$
\left.\frac{d}{d t} \mathcal{A}\left(F_{t \#} V\right)\right|_{t=0}=\int_{M} \operatorname{div}_{M} X(x) \theta(x) d \mathcal{H}^{d}(x)=\int_{\Omega \times G_{d, n}} \operatorname{div}_{T} X(x) d V(x, T)
$$

with $F_{t}:(x, P) \mapsto\left(\phi_{t}(x), d_{x} \phi_{t}(P)\right)$.

Définition 2.3. A $d$-varifold on $\Omega \subset \mathbb{R}^{n}$ is stationary if for every $X \in C_{c}^{1}\left(\Omega, \mathbb{R}^{n}\right)$,

$$
\int_{\Omega \times G_{d, n}} \operatorname{div}_{T} X(x) d V(x, T)=0
$$

Définition 2.4. If $V=v(M, \theta)$ is a $d$-rectifiable varifold, the measure $\theta \mathcal{H}_{\mid M}^{d}$ is called the weight of $V$ and denoted $\|V\|$. For a general varifold $V$, the weight of $V$ is the positive measure defined by $\|V\|(B)=V\left(\pi^{-1}(B)\right)$ for every $B \subset \Omega$ Borel, with $\left\{\begin{aligned} \pi: \Omega \times G_{d, n} & \rightarrow \Omega \\ (x, S) & \mapsto x\end{aligned}\right.$

\subsection{First variation of a varifold}

Because of the previous computations, we understand the importance of the following linear functional:

Définition 2.5 (First variation of a varifold). The first variation of a $d$-varifold on $\Omega \subset \mathbb{R}^{n}$ is the linear functional

$$
\begin{aligned}
\delta V: C_{c}^{1}\left(\Omega, \mathbb{R}^{n}\right) & \rightarrow \mathbb{R} \\
X & \mapsto \int_{\Omega \times G_{d, n}} \operatorname{div}_{P} X(x) d V(x, P)
\end{aligned}
$$

Définition 2.6. We say that a $d$-varifold on $\Omega$ has locally bounded first variation when the linear form $\delta V$ is continuous that is to say, for every compact set $K \subset \Omega$, there is a constant $c_{K}$ such that for every $X \in C_{c}^{1}\left(\Omega, \mathbb{R}^{n}\right)$ with $\operatorname{supp} X \subset K$

$$
|\delta V(X)| \leq c_{K} \sup _{K}|X|
$$

Now, if a $d$-varifold $V$ has locally bounded first variation, the linear form $\delta V$ can be extended into a continuous linear form on $C_{c}\left(\Omega, \mathbb{R}^{n}\right)$ and then by Riesz Theorem, there exists a Radon measure on $\Omega$ (still denoted $\delta V$ ) such that

$$
\delta V(X)=\int_{\Omega} X \cdot \delta V \quad \text { for every } X \in C_{c}\left(\Omega, \mathbb{R}^{n}\right)
$$

Thanks to Radon-Nikodym Theorem, we can derive $\delta V$ with respect to $\|V\|$ and there exists a function $H \in$ $L_{l o c}^{1}(\Omega,\|V\|)$ and a measure $\delta V_{s}$ singular to $\|V\|$ such that

$$
\delta V=-H\|V\|+\delta V_{s}
$$

Définition 2.7. In the previous decomposition, $H$ is called the generalized mean curvature vector. 


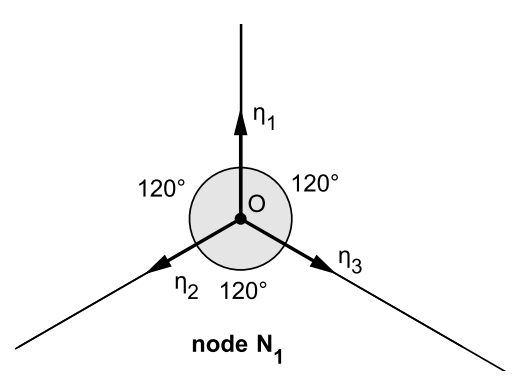

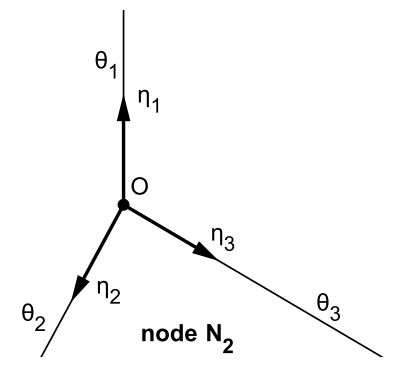

FigURE 3. Junctions

\subsection{Examples of computation of $\delta \mathrm{V}$}

Of course, for a smooth closed $d$-submanifold $M \subset \mathbb{R}^{n}$ and $V=v(M, 1)$ the varifold associated with multiplicity 1 , thanks to the divergence theorem, we have that $\delta V=H \mathcal{H}_{\mid M}^{d}$ with $H$ the mean curvature vector of $M$.

Exemple 2.8 (A line segment). We begin with a simple computation, let $M=[A B]$ be a line segment in $\mathbb{R}^{2}$ parametrized by $\gamma(t)=(1-t) a+t b$. Let $P$ denote the straight line underlying $[A B]$ and $\eta=\frac{\gamma^{\prime}(t)}{\left|\gamma^{\prime}(t)\right|}$. Let $V=v(M, 1)$ be the canonical 1 -varifold associated to $[A B]$ with multiplicity 1 . For every $X \in C_{c}^{1}\left(\mathbb{R}^{2}, \mathbb{R}^{2}\right)$,

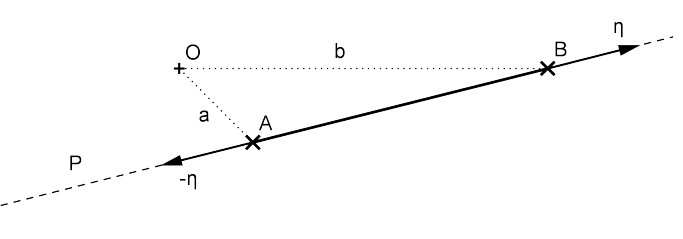

$$
\begin{aligned}
\delta V(X) & =\int_{M} \operatorname{div}_{M} X d \sigma=\int_{0}^{1} \operatorname{div}_{P} X(\gamma(t))\left|\gamma^{\prime}(t)\right| d t \\
& =\int_{0}^{1}<\nabla X(\gamma(t)), \eta>\cdot \eta\left|\gamma^{\prime}(t)\right| d t=\int_{0}^{1}<\nabla X(\gamma(t)), \underbrace{\left|\gamma^{\prime}(t)\right| \eta}_{\gamma^{\prime}(t)}\rangle \cdot \eta d t \\
& =\int_{0}^{1} \frac{d}{d t}(X(\gamma(t))) d t \cdot \eta=(X(b)-X(a)) \cdot \eta
\end{aligned}
$$

Thus $\delta V=\left(\delta_{b}-\delta_{a}\right) \eta$.

Exemple 2.9 (Curvature at a junction, Fig.3). We now consider two examples of triple junction, that is to say the union of three half-lines meeting at a same point $O$. In the first example, denoted by $V_{1}=v\left(N_{1}, 1\right)$, the angles between the three half-lines are all equal (to $\frac{\pi}{3}$ ) and the multiplicity is 1 everywhere while in the second example, denoted by $V_{2}=v\left(N_{2}, \theta\right)$, each half-line is directed by a unit vector $\eta_{i}$ with a multiplicity $\theta$ constant and equal to $\theta_{i}$ on each half-line $i=1,2,3$. A very important property of generalized curvature, coming from the definition itself, is linearity. Therefore, if we know the curvature of a half-line, we just have to make an addition to know the curvature of the whole junction. Thus we first compute (in a very similar way to the previous computation) the curvature of a half-line $W$ with unit direction vector $u$, constant multiplicity $\theta_{0}$ and parametrized by $\gamma(t)=t u$.

$$
\text { For } \begin{aligned}
X \in C_{c}^{1}\left(\mathbb{R}^{2}, \mathbb{R}^{2}\right), \quad \delta W(X) & =\int_{0}^{\infty}<\nabla X(\gamma(t)), u>\cdot u \theta_{0}\left|\gamma^{\prime}(t)\right| d t \\
& =\theta_{0} \int_{0}^{\infty} \frac{d}{d t}(X(\gamma(t))) d t \cdot u=-X(0) \cdot \theta_{0} u=\delta_{0}(X) \cdot \theta_{0} u
\end{aligned}
$$


Thus $\delta V_{1}=-\delta_{0} \cdot \underbrace{\left(\eta_{1}+\eta_{2}+\eta_{3}\right)}_{0}=0$ and more generally $\delta V_{2}=-\delta_{0} \cdot\left(\theta_{1} \eta_{1}+\theta_{2} \eta_{2}+\theta_{3} \eta_{3}\right)$.

\subsection{Orthogonality of the mean curvature}

It is well known that for a smooth submanifold $M$ of $\mathbb{R}^{n}$, the mean curvature vector is orthogonal to the tangent plane at every point. The first variation of the area functional tells us that the mean curvature vector points in the direction of increasing area. Now, considering a rectifiable varifold $V=v(M, \theta)$, area depends on the support $M$ and on the multiplicity $\theta$ so that if $\theta$ is varying, it produces a tangential mean curvature term.

Exemple 2.10 (Multiplicity generating curvature). Consider the straight line $D=\{y=0\} \subset \mathbb{R}^{2}$ with the multiplicity function $\theta(x, y)=x^{2}+1$. We compute the generalized curvature of the varifold $V=v(D, \theta)$ :

$$
\begin{aligned}
\delta V(X) & =\int_{D} \operatorname{div}_{D} X \theta d \mathcal{H}^{1}=\int_{\mathbb{R}} \operatorname{div}_{D} X(x, 0)\left(x^{2}+1\right) d x \\
& =\int_{\mathbb{R}} \partial_{1} X_{1}(x, 0)\left(x^{2}+1\right) d x=\underbrace{\left[X_{1}\left(x^{2}+1\right)\right]}_{0 \text { for } X \in C_{c}}-\int_{\mathbb{R}} X_{1}(x, 0) 2 x d x \\
& =-\int_{D} X(x, y) \cdot H(x, y) \theta(x, y) d \mathcal{H}^{1}(x, y)
\end{aligned}
$$

where $H(x, 0)=\left(\frac{2 x}{x^{2}+1}, 0\right)$ is tangent to $\mathrm{D}$.

Nevertheless, if $V$ is an integral varifold, that is to say $V$ is a rectifiable varifold with integer-valued multiplicity, Brakke has shown the following theorem of orthogonality (cf. [4])

Théorème 2.11 (Orthogonality of the mean curvature vector for integral varifolds). If $V=v(M, \theta)$ is an integral varifold with locally bounded first variation, if $\|V\|=\theta \mathcal{H}_{\mid M}^{d}$ and $\delta V=H\|V\|+\delta V_{s}$ with $\delta V_{s} \perp\|V\|$, then the mean curvature vector $H$ is orthogonal to the approximate tangent plane $T_{x} M$ for $\|V\|$ almost every point.

Remarque 2.12. In general, the singular part of the first variation can be much more complex than in the previous examples. See for instance [7] (p.33) for an example of varifold $V$ such that $\delta V_{s}$ is supported by a Cantor set.

\subsection{The hypothesis "of locally bounded first variation"}

We give an example of a varifold which has no locally bounded first variation. Exemple 2.13. Consider the straight line $D=\{y=0\} \subset \mathbb{R}^{2}$ with multiplicity 1 and a straight line $d$ not parallel to $D$, with unit direction vector $u=\left(u_{1}, u_{2}\right)$. If $V$ is the 1 -varifold $\mathcal{H}_{\mid D}^{1} \otimes \delta_{d}$, then $\delta V$ is not locally bounded. Indeed, take $\varepsilon>0$ and denote $D_{-\varepsilon}=\{y=-\varepsilon\}$ and $D_{\varepsilon}=\{y=\varepsilon\}$. Let $\beta \in C_{c}^{1}\left(\mathbb{R}^{2}, \mathbb{R}\right)$ be such that $\beta \equiv 1$ on $D_{\varepsilon}, \beta \equiv-1$ on $D_{-\varepsilon}$ and $\beta$ is linear between $D_{-\varepsilon}$ and $D_{\varepsilon}$ (at least for $-1 \leq x \leq 1$ ). Define $X_{\varepsilon}=\beta e_{2}$ (if $\left(e_{1}, e_{2}\right)$ is the canonical basis of $\mathbb{R}^{2}$ ), then one can compute for $x \in D$,

$$
\operatorname{div}_{d} X_{\varepsilon}(x)=\frac{1}{\varepsilon} u_{2}^{2}
$$

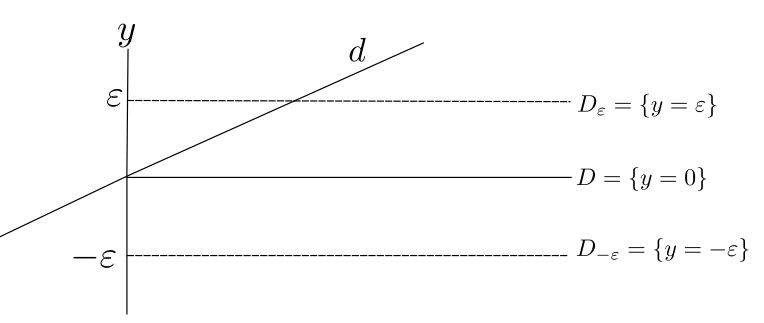

and $u_{2} \neq 0$ because $D$ and $d$ are not parallel. Hence $\delta V\left(X_{\varepsilon}\right) \geq \frac{1}{\varepsilon} u_{2}^{2} \int_{-1}^{1} d x=\frac{2}{\varepsilon} u_{2}^{2} \rightarrow+\infty$ when $\varepsilon \rightarrow 0$. 
ESAIM: PROCEEDINGS

\section{Rectifiability and Compactness Theorem}

\subsection{Rectifiability Theorem}

The following result gives an answer to the question: how can we know if a varifold is rectifiable? (see [8] Theorem 42.4 p. 243).

Théorème 3.1. Suppose that the varifold $V$ has locally bounded first variation in $\Omega \subset \mathbb{R}^{n}$ and $\theta^{d}(\|V\|, x)>0$ for $\|V\|$-almost every $x$. Then $V$ is a d-rectifiable varifold.

\subsection{Compactness Theorem}

The class of varifolds contains objects with weaker regularity than the class of submanifolds but this loss of regularity has the advantage that one can endow this class with a notion of convergence that brings compactness.

Définition 3.2 (Convergence in the sense of varifolds). We say that a sequence $\left(V_{i}\right)_{i}$ of $d$-varifolds converges to a $d$-varifold $V$ if $V_{i}$ converges weakly* to $V$ that is to say, for every $\varphi \in C_{c}\left(\Omega \times G_{d, n}, \mathbb{R}\right)$,

$$
\int \varphi d V_{i} \rightarrow \int \varphi d V
$$

Remarque 3.3. By defininition, convergence in the sense of varifolds implies convergence of the mass and convergence of the generalized curvature that is to say, if $V_{i} \rightarrow V$ weakly* then

(1) $\left\|V_{i}\right\|$ converges to $\|V\|$ weakly*,

(2) $\delta V_{i}$ converges to $\delta V$ weakly*.

Remarque 3.4. All results about convergence in the sense of Radon measures can be applied to the convergence in the sense of varifolds (think of semi-continuity properties for instance).

From the two previous remarks, it is possible to deduce the semi-continuity of the area functional:

Proposition 3.5. If $V_{i}$ converges to $V$ in the sense of varifolds then

$$
\|V\|(A) \leq \liminf _{i}\left\|V_{i}\right\|(A) \quad \text { for every } A \text { Borel }
$$

Théorème 3.6 (Allard Compactness Theorem). Let $\left(V_{j}\right)_{j}$ be a sequence of d-rectifiable varifolds with locally bounded first variation on an open set $\Omega \subset \mathbb{R}^{n}$ and such that $\theta_{j} \geq 1\left\|V_{j}\right\|$-almost everywhere. If

$$
\sup _{j}\left\{\left\|V_{j}(W)\right\|+\left|\delta V_{j}\right|(W)\right\} \leq c(W)<+\infty
$$

for every open set $W \subset \subset \Omega$, then there exists a subsequence $\left(V_{j_{n}}\right)_{n}$ converging weakly* to a d-rectifiable varifold $V$ with locally bounded first variation on $\Omega$ such that $\theta \geq 1$ and moreover

$$
|\delta V|(W) \leq \liminf _{n \rightarrow \infty}\left|\delta V_{j_{n}}\right|(W) \quad \forall W \subset \subset \Omega
$$

If for all $j, V_{j}$ is a integer-valued varifold then $V$ is integer-valued too.

Varifolds being endowed with a notion of convergence bringing compactness and a notion of generalized curvature and compactness property, they are well suited to tackle variational problems involving surfaces such as the minimization of the area functional or more generally functional involving area and curvature. The lower semi-conitnuity of the area functional has already been pointed out in Proposition 3.5, let us end with the lower semi-continuity of the Willmore functional: 
Définition 3.7. For a varifold $V$ in $\Omega$ of locally bounded first variation and such that $\delta V \ll\|V\|$ i.e. $\quad \delta V=H\|V\|$ with $H \in \mathrm{L}_{l o c}^{1}(\Omega,\|V\|)$, one defines the Willmore energy of $V$ by

$$
W(V)=\int_{\Omega}|H|^{p} d\|V\|
$$

The following semi-continuity result for the Willmore functional is a direct consequence of 2.36 in [3].

Théorème 3.8. Let $p>1$ and let $V_{n}$ be a sequence of varifolds converging weakly* to a varifold $V$. If for all $n, \delta V_{n} \ll\left\|V_{n}\right\|$ and

$$
\sup _{n} W\left(V_{n}\right)<+\infty
$$

then $\delta V \ll\|V\|$ and $W(V) \leq \liminf _{n} W\left(V_{n}\right)$

\section{REFERENCES}

[1] W. K. Allard. On the first variation of a varifold. The Annals of Mathematics, 95(3), 1972.

[2] F. Almgren. The theory of varifolds: A variational calculus in the large for the $k$-dimensional area integrand.

[3] L. Ambrosio, N. Fusco, and D. Pallara. Functions of Bounded Variation and Free Discontinuity Problems. Oxford University Press, 2000.

[4] K.A. Brakke. The motion of a surface by its mean curvature. Princeton University Press, 1978.

[5] J. Douglas. Solutions of the problem of plateau. Transactions of the American Mathematical Society, 1931.

[6] L.C. Evans and R.F. Gariepy. Measure Theory and Fine Properties of Functions. CRC Press, 1992.

[7] C. Mantegazza. Su alcune definizioni deboli di curvatura per insiemi non orientati. Tesi di Laurea, 1993. CVGMT http://cvgmt.sns.it/papers/man93/.

[8] Leon Simon. Lectures on Geometric Measure Theory. Centre for Mathematical Analysis, Australian National University, 1983. 